\title{
Epigenetic Identification of Mitogen-activated Protein Kinase 10 as a Functional Tumor Suppressor and Clinical Significance for Hepatocellular Carcinoma
}

\author{
Liping Tang ${ }^{1}$, Shasha Zhu ${ }^{2}$, Weiyan Peng ${ }^{3}$, Xuedong Yin ${ }^{3}$, Cui Tan ${ }^{3}$, Yaying Yang ${ }^{\text {Corresp. } 4}$ \\ ${ }^{1}$ Department of Gastroenterology, the First Affiliated Hospital of Chongqing Medical University, Chongqing Medical University, Chongqing, China \\ 2 The Center for Clinical Molecular Medical Detection, the First Affiliated Hospital of Chongqing Medical University, Chongqing, Chongqing, China \\ ${ }^{3}$ Chongqing Key Laboratory of Molecular Oncology and Epigenetics, the First Affiliated Hospital of Chongqing Medical University, Chongqing Medical \\ University, Chongqing, Chongqing, China \\ 4 Department of Pathology, Molecular Medicine and Cancer Research Center, Chongqing Medical University, Chongqing, China \\ Corresponding Author: Yaying Yang \\ Email address: 100500@cqmu.edu.cn
}

Background: Mitogen-activated protein kinase 10 (Mapk10) is a member of the c-jun Nterminal kinases (jnk) subgroup in the MAPK superfamily, and was proposed as a tumor suppressor inactivated epigenetically. Its role in hepatocellular carcinoma(HCC) has not yet been illustrated. We aimed to investigate the expression and epigenetic regulation of mapk10 as well as its clinical significance in HCC. Results: Mapk10 was expressed in almost all the normal tissues including liver, while we found that the protein expression of MAPK10 was significantly downregulated in clinical samples of HCC patients compared with these levels in adjacent normal tissues (29/46, $p<0.0001)$. Clinical significance of MAPK10 expression was then assessed in a cohort of 59 HCC cases, which indicated its negative expression was significantly correlated with advanced tumor stage $(p=0.001)$, more microsatellite nodules $(p=0.025)$, higher serum $\operatorname{AFP}(p=0.001)$ and shorter overall survival time of HCC patients. Methylation was further detected in $58 \%$ of the HCC cell lines we tested and in $66 \%$ of primary HCC tissues by methylation-specific PCR (MSP), which was proved to be correlated with the silenced or downregulated expression of mapk10. To get the mechanisms more clear, the transcriptional silencing of mapk10 was reversed by pharmacological demethylation, and ectopic expression of mapk10 in silenced HCC cell lines significantly inhibited the colony formation ability, induced apoptosis, or enhanced the chemosensitivity of HCC cells to 5-fluorouracil. Conclusion: Mapk10 appears to be a functional tumor suppressor gene frequently methylated in HCC, which could be a valuable biomarker or a new diagnosis and therapy target in a clinical setting. 
1 Epigenetic Identification of Mitogen-activated Protein Kinase 10 as a Functional Tumor 2 Suppressor and Clinical Significance for Hepatocellular Carcinoma

3

4

Liping Tang ${ }^{1}$, Shasha Zhu², Weiyan Peng ${ }^{3}$, Xuedong Yin ${ }^{3}$, Cui Tan ${ }^{3}$ Yaying Yang ${ }^{4}$

${ }^{1}$ Department of Gastroenterology, the First Affiliated Hospital of Chongqing Medical University, Chongqing Medical University, Chongqing, 400016, P.R. China.

${ }^{2}$ The Center for Clinical Molecular Medical Detection, the First Affiliated Hospital of Chongqing Medical University, Chongqing, 400016, P.R., China.

${ }^{3}$ Chongqing Key Laboratory of Molecular Oncology and Epigenetics, the First Affiliated Hospital of Chongqing Medical University, Chongqing Medical University, Chongqing,400016, P.R. China.

${ }^{4}$ Department of Pathology, Molecular Medicine and Cancer Research Center, Chongqing Medical University, Chongqing,400016, P.R. China

\section{E-maill:}

Liping Tang, 547053591@qq.com

Shasha Zhu,shasha0310@foxmail.com

Weiyan Peng,pwy16@sina.com

Xuedong Yin,13753950@qq.com

Cui Tan,tancui0803@163.com

Yaying Yang,100500@cqmu.edu.cn

\section{*Corresponding Author:}

Yaying Yang

Department of Pathology, Molecular Medicine and Cancer Research Center, Chongqing Medical University, Chongqing,400016, P.R. China

Tel: +86- 23-68485789

E-mail: 100500@cqmu.edu.cn 


\section{Abstract}

Background: Mitogen-activated protein kinase 10 (Mapk10) is a member of the c-jun Nterminal kinases (Jnk) subgroup in the MAPK superfamily, and was proposed as a tumor suppressor inactivated epigenetically. Its role in hepatocellular carcinoma (HCC) has not yet been illustrated. We aimed to investigate the expression and epigenetic regulation of mapk10 as well as its clinical significance in HCC.

Results: Mapk10 was expressed in almost all the normal tissues including liver, while we found that the protein expression of MAPK10 was significantly downregulated in clinical samples of HCC patients compared with these levels in adjacent normal tissues (29/46, $p<0.0001)$. Clinical significance of MAPK10 expression was then assessed in a cohort of 59 HCC cases, which indicated its negative expression was significantly correlated with advanced tumor stage $(p=0.001)$, more microsatellite nodules $(p=0.025)$, higher serum $\operatorname{AFP}(p=0.001)$ and shorter overall survival time of HCC patients. Methylation was further detected in $58 \%$ of the HCC cell lines we tested and in $66 \%$ of primary HCC tissues by methylation-specific PCR (MSP), which was proved to be correlated with the silenced or downregulated expression of mapk10. To get the mechanisms more clear, the transcriptional silencing of mapk10 was reversed by pharmacological demethylation, and ectopic expression of mapk10 in silenced HCC cell lines significantly inhibited the colony formation ability, induced apoptosis, or enhanced the chemosensitivity of HCC cells to 5-fluorouracil.

Conclusion: Mapk10 appears to be a functional tumor suppressor gene frequently methylated in HCC, which could be a valuable biomarker or a new diagnosis and therapy target in a clinical setting.

Keywords: hepatocellular carcinoma, mapk10, tumor suppressor gene, methylation 


\section{Background}

Hepatocellular carcinoma (HCC) is one of the most lethal malignancies in humans. It is the sixth most common cancer worldwide and the leading cause of deaths among patients with cirrhosis in Europe and the USA accompanied about 782000 deaths per year ${ }^{1,2}$. Since the patients with HCC are always diagnosed at the advanced stage and the efficacy of curative surgery or adjuvant therapy including systemic chemotherapy on advanced HCC remains poor ${ }^{3-5}$, it is particularly important to find reliable biomarkers in $\mathrm{HCC}$ early diagnosis and potential therapeutic targets .

Genetic and epigenetic alterations in regulatory genes are critical in the development of HCC, resulting in the imbalance between oncogenes and tumor suppressor genes (TSG) ${ }^{6,7}$. For example, promoter $\mathrm{CpG}$ methylation, one of the epigenetic alterations, which occurs frequently during tumor development and progression, causes the loss of TSG functions. Accumulating evidence demonstrated that the promoter of $\mathrm{CpG}$ methylation was essential in initiating carcinogenesis and the epigenomic changes, and thus, could be applied as a new biomarker for the diagnosis, in clinical trials of epigenetic drugs, and as an add-on to the drugs such as multikinases $^{8-11}$. Furthermore, it was identified some valuable TSGs that were frequently methylated in HCC and established a specific methylation profile that would provide novel reliable biomarkers for HCC management ${ }^{12}$.

MAPK10 (JNK3) encoded by the mapk10 gene is a member of JNKs, which were implicated in multiple signaling pathways including apoptosis, differentiation, and proliferation ${ }^{13}$, 14. Previous studies showed that JNK contributed to the proliferation or survival of tumors; nevertheless, it might also act as a suppressor in some tumor cell types, indicating various roles of $\mathrm{JNK}$ in tumor progression ${ }^{15}$. Hitherto, the genetic and mechanistic basis for the complex role of JNK in tumors is yet to be elucidated.

Loss of heterozygosity $(\mathrm{LOH})$ at specific chromosomal regions in human tumors suggests the presence of candidate TSGs in the affected region. A deletion at 4q21.3 locus was identified in two esophageal carcinoma cell lines by high-resolution $(1 \mathrm{Mb})$ array-comparative genomic hybridization $(\mathrm{CGH})$; as a result, mapk10 was found to be localized within this deleted region ${ }^{16}$. Other reports demonstrated that the frequent loss of expression of mapk10 in 10/19 human cell lines originated in brain tumors and the JNK3 signaling pathway mediated cell apoptosis in the central nervous system in jnk3-deficient mice ${ }^{17,}{ }^{18}$. Moreover, the effect of Jnk deficiency on Ras-stimulated transformation resulted in a substantial increase in the number and growth of tumor nodules in vivo ${ }^{19}$, which was consistent with the conclusion that mapk10 plays a role in tumor suppression. Furthermore, previous studies showed that the expression of mapk10 was frequently downregulated or silenced in several tumor cell lines such as lymphoma, breast cancer, gastric cancer, in which, the methylation of mapk10 promoter is correlated with its expression ${ }^{20}$. Also, the ectopic expression of mapk10 markedly suppresses proliferation of breast cancer cells ${ }^{16}$.In conclusion, these results suggested that mapk10 might function as a TSG to suppress the tumorigenesis with respect to epigenetics. 
As far as we know, expression and role of mapk10 in HCC have not been illustrated. In our experiment, the expression of mapk10 was observed downregulated in HCC tissues, which dramatically correlated with the clinical characteristics and the overall survival (OS) of HCC patients. Furthermore, the experiments showed that promoter methylation was responsible for the mapk10 downregulation in HCC cell lines and tumor tissues, and the pharmacological demethylation and ectopic expression of mapk10 in silenced HCC cells could suppress the clonogenicity, induce the cell apoptosis, or enhance the sensitivity of HCC to 5fluorouracil (5-FU). Overall our finding indicated that mapk10 be a functional tumor suppressor gene frequently methylated in HCC, suggesting that new therapy targeting mapk10 holds promise for HCC patients.

\section{Materials and methods} Tumor cell lines

A series of HCC cell lines (Hep3B, HepG2, huH1, huH4, huH6, huH7, PLC/PRF/5, SNU387, SNU398, SNU423, SNU449, and SNU475) were maintained in Cancer Epigenetics laboratory, Department of Clinical Oncology, PWH, The Chinese University of Hong Kong, Shatin, Hong Kong and cultured in RPMI or DMEM (Gibco, USA) supplemented with $10 \%$ fetal bovine serum (FBS) (Gibco, USA) and incubated at $37{ }^{\circ} \mathrm{C}$ in a humidified chamber containing $5 \% \mathrm{CO}_{2}$.

\section{Tissues of HCC}

A total of 18 fresh primary $\mathrm{HCC}$ tissues were collected from patients who underwent hepatectomy for HCC in 2009 at the First Affiliated Hospital of Chongqing Medical University (Chongqing, China). Among them, 10 has the matched non-tumor liver samples, obtained from at least $5 \mathrm{~cm}$ distant from the tumor. All of the fresh tissues were processed routinely for histopathological assessment and immediately immersed in liquid nitrogen after surgical resection.

Along with the paraffin-embedded tissues, a retrospective cohort of 59 patients with HCC was enrolled in the current study. These patients underwent hepatectomy at the First affiliated hospital of Chongqing Medical University (Chongqing, China) between June 2005 and December 2009. All patients were diagnosed with primary HCC by two pathological doctors, and there was no previous radio- or chemotherapy before surgery. The study was carried out in accordance with the Declaration of Helsinki and the protocol was approved by Ethics Committee of Chongqing Medical University (Chongqing, China) and written informed consent was obtained from each patient involved in the study.

\section{Immunohistochemistry (IHC)}

IHC staining was carried out by using Ultrasensitive TMS-P (MXB, China). The primary 
antibody against MAPK10 was obtained from Abcam (Abcam, USA) and incubated the sections at a dilution 1:100. The staining intensity was scored as 0 (absent expression), 1(weak expression), 2 (moderate expression), and 3(strong expression) and the percentage of positive staining as $0(0 \%-9 \%), 1(10 \%-25 \%), 2(26 \%-50 \%)$ or $3(51 \%-100 \%)^{21}$. The final score was obtained by multiplying as the following: the staining intensity score $\times$ the percentage of positive staining and ranked from 0 to 9. So, the Mapk10 expression of HCC was defined as "","+","++"and"+++"corresponding to score 0, 1-3, 4-6 and 7-9 respectively. For further clinical analysis, we divided the HCC into two subgroups: the "Mapk10-negative" subgroup ("-") or "Mapk10-positive" subgroup"+-+++". The IHC staining score was performed in a double-blind manner by two pathologists to minimize the observational bias.

\section{Semiquantitative RT-PCR}

RT-PCR was conducted to detect the expression of Mapk10 in HCC cell lines. RT-PCR was performed for 32 cycles with hot-start Go-Taq (Promega, USA) ${ }^{22}$. The primers are listed in Supplementary Table 1.

\section{Western blot}

Primary antibodies against Mapk10 (1:000, Abcam, USA) and PARP ((1:000, Abcam, USA) were used. At $48 \mathrm{~h}$ post-transfection, the cells were collected and lysed in RIPA lysis buffer (Beyotime, China). Total protein was dissociated by SDS-PAGE, transferred onto PVDF membrane, and immunoblotted. The immunoreactive bands were exposed by the ECL detection system.

\section{Methylation-specific PCR (MSP)}

After DNA bisulfite treatment, MSP was performed in the HCC cell lines as well as in the primary HCC tumors and adjacent non-tumor tissues as described previously ${ }^{22}$. The MSP primers detecting the methylated or unmethylated alleles are MAPK10m3/MAPK10m5 and MAPK10u3/MAPK10u5, respectively (Supplementary Table 1) ${ }^{23}$. These primer pairs have been tested previously that they did not amplify any unbisulfited DNA. MSP was performed for 40 cycles with AmpliTaq-Gold (Applied Biosystems, USA) ${ }^{22}$. YccB1, a cell line of breast carcinoma with hypermethylated MAPK10 which has been demonstrated in the previous literature was used as the positive control of MAPK10 methylation ${ }^{23}$.

\section{5-aza-2'-deoxycytidine (Aza) and Trichostatin A (TSA) treatment}

A density of $1 \times 10^{5} \mathrm{HCC}$ cells $/ \mathrm{mL}$ were allowed to grow overnight. Then, fresh medium containing Aza at a final concentration of $10 \mathrm{mM}$ (Sigma-Aldrich, USA) was used to replace the culture medium. The cells were treated with Aza for $72 \mathrm{~h}$, followed by the histone deacetylase inhibitor TSA at $100 \mathrm{nM}$ for another $24 \mathrm{~h}$. Finally, the cells were collected for methylationspecific PCR (MSP) and the gray value of bands was analyzed by Image J software. 


\section{Gene cloning and plasmid construction}

The Mapk10 recombinant plasmids were purchased from GeneCopoeia (USA). These were termed as OmicsLink ${ }^{\mathrm{TM}}$ expression clone (EX-A1150-M29) and pcDNA3.0(+)-Flag-MAPK with the accurately confirmed sequence and orientation.

\section{Subcellular localization}

A density of $5 \times 10^{4}$ of Hep3B or HepG2 cells were planted on coverslips in a 6-well plate. Then, the cells were transfected with pcDNA3.0(+)-Flag-Mapk10 using Lipofectamine 2000 (Invitrogen, USA). After 24h, the cells were fixed with 4\% paraformaldehyde phosphate buffer solution and incubated with anti-Flag M2 monoclonal antibody (Sigma, USA), followed by FITC-conjugated rabbit anti-mouse IgG F(ab)2 antibody (Sigma, USA). Hereafter, cell nuclei were stained with DAPI (Thermo, USA) and images were captured by Leica TCS SP2 AOBS confocal laser-scanning microscope (Mannheim, Germany).

\section{Colony formation assay}

$1 \times 10^{5} \mathrm{Hep} 3 \mathrm{~B}$ and HepG2 cells/well were seeded in a 12-well plate and transfected with Mapk10 plasmid or control vector, using Lipofectamine 2000. After $48 \mathrm{~h}$, the cells were seeded into a 6-well plate, and subjected to G418 $(0.4 \mathrm{mg} / \mathrm{mL})$ for $10-14$ days, with the medium refreshed every 2 days. The surviving colonies were enumerated after Giemsa staining. All the experiments were conducted three times in triplicate wells.

\section{Apoptosis assay}

The cell apoptosis and viability were evaluated using the Annexin V-PE Apoptosis Detection Kit I (BD Biosciences, USA) by flow cytometry. Apoptosis was always graded as viable, early apoptotic, necrotic and late apoptotic, which corresponds to Annexin V-/7-AAD-, Annexin V+/ 7-AAD-,Annexin V-/7-AAD+ and AnnexinV+/7-AAD+. Hep3B and HepG2 cells were transfected with Mapk10 plasmid or the control vector and harvested after $48 \mathrm{~h}$. The GFP-positive cells were selected, and the apoptotic status was assessed by Annexin V-PE and 7AAD staining. Both early and late apoptotic cells were considered for relative apoptotic alterations. Apoptosis was also depended on the terminal deoxynucleotidyl transferase (TDT)mediated dUTP-digoxigenin nick end labeling (TUNEL) assay. At $48 \mathrm{~h}$ post-transfection, the apoptotic cells were detected using the In Situ Cell Death Detection Kit POD (Roche, USA), wherein the DNA fragmentation was determined and visualized by a fluorescence microscope. All the experiments were repeated 3 times.

\section{XTT proliferation assay}

HCC is resistant to systemic chemotherapy and 5-FU is one of the most common drugs for HCC. Herein, we assessed whether Mapk10 could enhance the chemotherapeutic effects of 5-FU on HCC cells. XTT Cell Proliferation Assay kit (Beyotime, China) was used to determine the chemotherapy-induced cytotoxicity on HCC cells. Mapk10 or control vector was transfected into HCC cells, respectively and treated with 5 -FU at different concentrations $(0,6.25,12.5,25,50$, 100 , and $200 \mu \mathrm{g} / \mathrm{mL}$ ) for $72 \mathrm{~h}$. Subsequently, the relative number of viable cells between 
213 Mapk10-transfected group and control vector-transfected group was compared and expressed as 214 a percentage using the following formula: cell viability $(\%)=$ A450 of cells treated with 5215 FU/A450 of cells without 5-FU treatment. Three independent experiments were performed, each 216 in duplicate.

\section{Statistical analysis}

The clinicopathological features in MAPK10-positive patients and MAPK10-negative patients were compared using the Pearson's chi-squared test. Kaplan-Meier plots and log-rank tests were used for the survival analysis. Student's t-test was used to compare the differences in the effect of MAPK10 expression on colony formation, cell apoptosis, and cell proliferation. SPSS 19.0 was used for data analysis. A p-value $<0.05$ was deemed as statistically significant.

\section{Results}

The protein expression of MAPK10 in clinical samples of HCC and its clinical significance in HCC patients

The cohort of 59 patients (Table 1) consisted of $77.9 \%$ males with a median age of 49.94 (range, 21-77) years. Chronic HBV carriers accounted for the majority (66.2\%). The median AFP ( $\alpha$-fetoprotein) value was 5181.8169 (range 2.8-96401) ng/ml. Among all the samples, 46 pairs were eligible for the comparison of the expression of mapk10 between HCC and adjacent non-tumor tissues by IHC. All of the 59 patients with follow-up data were well qualified for the clinical correlation analysis and OS analysis, with the median follow-up for 13.0 (range 2-46) months.

As for 46 paired paraffin-embedded tissues, all of the adjacent non-tumor tissues show the positive expression of MAPK10, whereas 29 HCC tissues show the negative expression, the negative expression rate of MAPK10 between the HCC and the adjacent non-tumor tissues was significant $(P<0.0001)$ (Figure 1). The clinicopathological correlation analysis showed that the negative expression of MAPK10 in HCC was significantly associated with higher serum AFP $(\mathrm{p}=0.05)$, more microsatellite nodules $(P=0.025)$ and advanced tumor stage $(P=0.001)$ (table 1$)$.

\section{Downregulated MAPK10 expression may indicate poor prognosis in HCC patients}

Prognostic significance of MAPK10 in HCC was also studied in this cohort of 59 patients with follow-up data, which indicated that the expression of MAPK10 was extremely correlative with OS of patients ( $\log$ rank=7.123, $P=0.008)$ : the median OS time in MAPK10-negative subgroup $(n=23)$ and MAPK10-positive subgroup $(n=36)$ were 10.86 months $(95 \%$ CI 5.927 to 15.792) and 24.040 months (95\% CI 17.511 to 30.568) respectively (Figure 2). These data suggested that MAPK10 could be an indicator for the prognosis of HCC patients.

\section{Expression profiling of Mapk10 in HCC cell lines}

Previous studies found a deletion at 4q21.3 in two esophageal carcinoma cell lines and found that Mapk10 was located in this deleted region with a 633bp bidirectional promoter, which was a typical $\mathrm{CpG}$ island, as well as that Mapk10 was expressed in all normal adult tissues ${ }^{23}$. However, 
254 semi-quantitative RT-PCR in our experiments revealed a frequently reduced or silenced 255 expression of Mapk10 in HCC cell lines (67\%, 8/12) (Figure 3). Together, the above results 256 showed that Mapk10 is a widely expressed gene but frequently disrupted in multiple HCC cell 257 lines.

\section{Frequent inactivation of Mapk10 by promoter CpG methylation}

In fresh primary tumors, the Mapk10 promoter was frequently methylated in $12 / 18(66 \%)$ HCC tissues, while reduced or no methylation was detected in the adjacent non-tumor tissues (Figure 4A), suggesting that the methylation of Mapk10 is tumor-specific. Furthermore, we detected Mapk10 methylation in HCC cell lines using MSP and found that the methylation of Mapk10 is associated with the dysregulated or silenced expression of the gene shown in RT-PCR (Figure 4B).

\section{Pharmacological and genetic demethylation restores Mapk10 expression}

Two HCC cell lines, Hep3B and HepG2, were subjected with DNA methyltransferase inhibitor Aza combined with histone deacetylase inhibitor Trichostatin A (TSA). The treatment restored the Mapk10 expression, accompanied by decreased methylated promoter alleles (HepG2) or increased unmethylated alleles (Hep3B) (Figure 5). The above results indicated that promoter methylation directly mediated the transcriptional silencing of Mapk10.

The frequent silencing of Mapk10 by hypermethylation in HCC cell lines rendered Mapk10 as a putative tumor suppressor. Furthermore, we evaluated the growth characteristics of the cells overexpressing Mapk10 by colony formation assay. HepG2 and Hep3B cell lines with silenced Mapk10 were transfected with Mapk10 recombinant plasmids, and the number of colonies was enumerated after 10-14-day culture under G418 selection. The subcellular localization of MAPK10 was examined by indirect immunofluorescence staining, which indicated that Flagtagged MAPK10 was localized in the nucleus after the transfection into HCC cells (Figure 6A) and the western blot detection of MAPK10 protein expression also confirmed the success of transfection (Figure 6B and 6C). The ectopic expression of Mapk10 markedly decreased the colony formation efficiency of Hep3B and HepG2 cells (34\% and 40\% respectively, $\left.{ }^{*} \mathrm{p}<0.01\right)$, indicating the growth inhibitory activity of Mapk10 (Figure 6D and 6E).

\section{Ectopic expression of Mapk10 induces apoptosis}

To explore the mechanism of the tumor suppression by Mapk10, we performed apoptosis assay using Annexin V-PE and 7-AAD double staining. The percentage of Annexin V (+/-) and 7-AAD (+/-) cells in GFP-positive cells by flow cytometry was determined. In Hep3B cells and HepG2 cells, the ectopic Mapk10 expression resulted in a significant increase in the number of apoptotic cells as compared to the control $(P<0.05)$ (Figure 7A). Furthermore, the apoptotic induction was assessed by TUNEL assay at the individual cell level. Green fluorescent signals, characteristic of cell apoptosis, were presented in Mapk10-transfected cells but less in control vector- transfected cells (Figure 7B). Increased protein level of cleaved-PARP was also shown in HepG2 and Hep3B cells after MAPK10 transduction (Figure 7C). 
295

296

297

298

299

300

301

302

303

304

305

306

307

308

309

310

311

312

313

314

315

316

317

318

319

320

321

322

323

324

325

326

327

328

329

330

331

332

\section{Mapk10 enhances the chemosensitivity of HCC cells to 5-FU}

The efficacy of chemotherapeutic drugs is dependent on their ability to trigger apoptosis ${ }^{21}$. Since our studies showed that Mapk10 induces apoptosis in HCC cells, we speculated the enhanced chemotherapy effects of 5-FU on HCC cells. Notably, the cell viability in Mapk10transfected group was significantly inhibited as compared to that in the control group (Figure 8A). Furthermore, the IC50 of HepG2 cells for 5-FU was significantly reduced after Mapk10 transfection, which was $16.253 \mu \mathrm{g} / \mathrm{mL}$ in the Mapk10-transfected group as compared to 251.356 $\mu \mathrm{g} / \mathrm{mL}$ for the control group (Figure $8 \mathrm{~B}$ ).

Figure 8 MAPK10 enhances the chemosensitivity of HCC cells to 5-FU. A. Cell viability assay of Hep3B after transfected with Mapk10. B. Cell viability assay of HepG2 after transfected with Mapk10. $\left({ }^{*} P<0.01,{ }^{* *} P<0.01\right)$

\section{Discussion}

In the present study, we found that the expression of Mapk10 is frequently silenced or downregulated in most of the paraffin-embedded HCC tissues $(63 \%)$ when compared with the adjacent non-tumor tissues. The clinical analysis of the paraffin-embedded tissues also revealed that the negative expression of Mapk10 was significantly associated with higher serum AFP, more tumor microsatellite nodules, advanced tumor stage, and the shorter OS of HCC patients. Moreover, hypermethylation of Mapk10 promoter was further detected in most of these primary HCC tumors $(66 \%)$, and it was also demonstrated that the hypermethylation is correlated with the silenced gene expression of Mapk10 in several HCC cell lines. Conversely, Mapk10 hypermethylation was reduced in paired non-tumor tissues, suggesting a role of Mapk10 as a candidate TSG in the pathogenesis of HCC. The expression of reactivated Mapk10 could be observed after treatment with the demethylating reagent in silenced HCC cells, indicating that DNA hypermethylation directly mediated the inactivation of Mapk10. Furthermore, the ectopic expression of Mapk10 in silenced HCC cells dramatically inhibited the tumor cell colony formation, induced the cell apoptosis, and enhanced the chemosensitivity of HCC cells to 5-FU. Thus, Mapk10 was suggested to function as a TSG inactivated epigenetically for HCC and might act as a clinical biomarker for early diagnosis and therapy target.

The epigenetic inactivation of TSGs by the hypermethylation of promoter has been recognized as an alternative and critical mechanism in tumorigenesis. Rather than changing the genetic information, DNA methylation only changes the readability of the DNA and results in the inactivation of genes by subsequent suppression of mRNA transcription. As we know, TSG protects the cells from malignant transformation by mechanisms such as apoptosis or cell cycle regulation ${ }^{24}$. Thus, the aberrant hypermethylation of the promoter and epigenetic silencing of

TSG affect every step of tumor progression ${ }^{25-27}$. Currently, the transcriptional silencing of TSGs associated with DNA methylation has been shown to be a common epigenetic event in several 
333

334

335

336

337

338

339

340

341

342

343

344

345

346

347

348

349

350

351

352

353

354

355

356

357

358

359

360

361

362

363

364

365

tumors, including hematologic malignancies, nasopharyngeal carcinomas, gastroenterological neoplasia, esophageal carcinomas, and breast cancers ${ }^{25,}{ }^{28-31}$. The identification of potential TSGs and specific methylation profiles of TSGs for every specific tumor might be valuable for the diagnosis of tumor and prediction of the prognosis ${ }^{32}$.

So far, the studies in HCC found some epigenetic silenced TSGs, such as p16, E-cadherin, hMLH1, SOCS1, SOCS, and RASSF1A, which play a role in proliferation, apoptosis, cell adhesion, invasion, and DNA repair ${ }^{7}$. Compared to several other tumors, the prognosis of HCC is poor, and the underlying molecular pathogenesis, including the epigenetic mechanism, is less understood. Most of the HCCs arise on the background of chronic liver disease and chronic hepatitis B virus (HBV), hepatitis C virus (HCV), aflatoxin, alcohol consumption, and hemochromatosis constitute the major factors associated within $\mathrm{HCC}$ initiation ${ }^{33}$. Reportedly, the aberrant methylation of TSG in HCC occurs in a genetic- and disease-specific manner and could be observed not only in advanced tumors but also in premalignant conditions of $\mathrm{HCC}^{7}, 12$. Also, studies revealed that $\mathrm{HCC}$ and precancerous lesions might present epigenetic signals correlated with the specific risk factors and tumor progression stage, indicating that the epigenetic changes in HCC might be potential targets for biomarker discovery or future therapeutic strategies ${ }^{34}$.

Mapk10 (JNK3) is a third member of JNKs, which belongs to MAP kinases, implicated in critical physiological processes, including proliferation, apoptosis, and differentiation ${ }^{14}, 15$. Numerous studies have confirmed that JNK acts as a suppressor of Ras transformation and contributes towards apoptosis in tumors. For example, the activation of the mitochondrial apoptotic pathway in many cell types and the interferon-alpha (IFN- $\alpha$ )-induced apoptosis in Bcell lymphoma were reported ${ }^{35}$. Nevertheless, it might also enhance the proliferation and survival responses of some tumors ${ }^{15}$, thereby rendering it as a putative TSG. Thus, the strategies of tumor prevention and therapy necessitate further in-depth investigation. Unlike $J N K 1$ and $J N K 2$, Mapk10 (JNK3) is not expressed ubiquitously and performs non-redundant functions ${ }^{18}$. Frequent loss of Mapk10 expression has been detected in a panel of tumor cell lines ${ }^{16,17}$, together with the proapoptotic functions observed ${ }^{18,19}$, suggesting its potential role as a TSG. Previously, it was identified that Mapk10 (JNK3) could be inactivated in multiple lymphomas and carcinomas by epigenetic methylation, albeit only few studies have addressed this concern ${ }^{16}$. In the present study, we focused on HCC. Mapk10 (JNK3) was shown to be silenced or downregulated in a majority of the HCC cell lines or primary HCC tumor samples examined, while the demethylating reagent could restore its expression in silenced HCC cells. Further steps indicated that the ectopic expression of Mapk10 in silenced HCC cells significantly inhibited the 
tumor cell growth or induced the apoptosis. So our results demonstrated that Mapk10 served as a TSG in HCC and could be inactivated by promoter methylation in tumorigenesis, which might be one of the mechanisms in HCC progression. However, due to the complexity of gene expression regulation, the additional mechanisms beside methylation may be involved in the regulation of Mapk10 expression and needed for further explored.

As we know, chemoresistance was facing challenge with respect to the treatment of HCC patients ${ }^{36}$. The efficacy of chemotherapeutic drugs has been speculated to depend on the ability to trigger apoptosis ${ }^{37}$. In our research, Since MAPK10 could induce apoptosis in these HCC cells; we speculated that it might also enhance the efficacy of the chemotherapeutic drugs on HCC. Herein, the chemotoxic effects of 5-FU on HCC cells were compared between the Mapk10transfected group and the control vector-transfected group. Consequently, significant enhancement of chemosensitivity of HCC cells to 5-FU was observed in Mapk10-transfected group, indicating ectopic expression of Mapk10 as a novel therapeutic assistant for drug resistance in HCC. Finally, the clinical correlation analysis found that the silenced expression of Mapk10 in HCC was dramatically correlative with the advanced tumor stage and poor prognosis of HCC patients. This phenomenon supported that Mapk10 expression might be a valuable molecular biomarker for HCC in a clinical setting.

\section{Conclusion}

In summary, Mapk10 is functions as a tumor suppressor and frequently inactivated by promoter methylation in HCC. The downregulated expression of Mapk10 caused by hypermethylation in HCC tumors is correlated with the advanced tumor stages and poor prognosis of patients, which hinted that the epigenetic inactivation of Mapk10 is one of the major mechanisms in tumorigenesis of HCC, along with the evidence that Mapk10 is a general TSG for HCC. The high incidence of epigenetic inactivation of Mapk10 in HCC rendered hypermethylation as a reliable molecular biomarker and a potential epigenetic therapeutic target in HCC management.

\section{Declaration}

\section{Ethics approval and consent to participate}

Not applicable.

\section{Consent for publication}

Not applicable.

\section{Competing interests}

The authors have declared that there were no competing interests.

\section{Funding}

This work was supported by the National Natural Science Foundation Committee of China under contract No. 81000907.

\section{Authors' contributions}


407 Liping Tang and Shasha Zhu carried out the studies, participated in collecting data, and drafted 408 the manuscript. Weiyan Peng and Xuedong Yin performed the statistical analysis and 409 participated in its design. Cui Tang and Yaying Yang participated in acquisition, analysis, or 410 interpretation of data and draft the manuscript. All authors read and approved the final 411 manuscript.

412 Acknowledgements

413 We greatly thank Professor Qian Tao (Cancer Center, Department of Clinical Oncology, PWH, 414 The Chinese University of Hong Kong, Shatin, Hong Kong) for his kindly help and guidance in 415 the experimental design and methodology. 


\section{References:}

417 1. Lombardi A, Grimaldi A, Zappavigna S, Misso G, Caraglia M. Hepatocarcinoma: genetic and epigenetic features. Minerva Gastroenterol Dietol. 2018;64(1):14-27.

2. Bray F, Ferlay J, Soerjomataram I, Siegel RL, Torre LA, Jemal A. Global cancer statistics 2018: GLOBOCAN estimates of incidence and mortality worldwide for 36 cancers in 185 countries. CA Cancer J Clin. 2018;68(6):394424.

3. J Z, A V, JC N, JM L. Genetic Landscape and Biomarkers of Hepatocellular Carcinoma. Gastroenterology. 2015;149(5):1226-1239.

4. J C, R J, J Z, et al. Potential molecular, cellular and microenvironmental mechanism of sorafenib resistance in hepatocellular carcinoma. Cancer Lett. 2015;367(1):1-11.

5. Kudo M, Trevisani F, Abou-Alfa GK, Rimassa L. Hepatocellular Carcinoma: Therapeutic Guidelines and Medical Treatment. Liver Cancer. 2017:16-26.

6. Lee SM, Kim-Ha J, Choi WY, et al. Interplay of genetic and epigenetic alterations in hepatocellular carcinoma. Epigenomics-Uk. 2016;8(7):993-1005.

7. Tischoff I, Tannapfe A. DNA methylation in hepatocellular carcinoma. World $J$ Gastroenterol. 2008;14(11):1741-1748.

8. Nakamura M, Chiba T, Kanayama K, et al. Epigenetic dysregulation in hepatocellular carcinoma: an up-to-date review. Hepatol Res. 2019;49(1):3-13.

9. Hardy T, Mann DA. Epigenetics in liver disease: from biology to therapeutics. Gut. 2016;65(11):1895-1905.

10. Ozen C, Yildiz G, Dagcan AT, et al. Genetics and epigenetics of liver cancer. N Biotechnol. 2013;30(4):381384.

11. Sceusi EL, Loose DS, Wray CJ. Clinical implications of DNA methylation in hepatocellular carcinoma. $H P B$ (Oxford). 2011;13(6):369-376.

12. Nishida N, Nagasaka T, Nishimura T, Ikai I, Boland CR, Goel A. Aberrant methylation of multiple tumor suppressor genes in aging liver, chronic hepatitis, and hepatocellular carcinoma. Hepatology. 2008;47(3):908-918.

13. Zhang Y, Dong C. Regulatory mechanisms of mitogen-activated kinase signaling. Cell Mol Life Sci. 2007;64(21):2771-2789.

14. Davis RJ. Signal transduction by the JNK group of MAP kinases. Cell. 2000;103(2):239-252.

15. Kennedy NJ, Davis RJ. Role of JNK in tumor development. Cell Cycle. 2003;2(3):199-201.

16. Ying J, Li H, Cui Y, Wong AH, Langford C, Tao Q. Epigenetic disruption of two proapoptotic genes MAPK10/JNK3 and PTPN13/FAP-1 in multiple lymphomas and carcinomas through hypermethylation of a common bidirectional promoter. Leukemia. 2006;20(6):1173-1175. chromosomal assignment, and loss of expression in brain tumors. J Hum Genet. 2001;46(4):182-187. mice lacking the Jnk3 gene. Nature. 1997;389(6653):865-870. transformation by the JNK signal transduction pathway. Genes Dev. 2003;17(5):629-637. common bidirectional promoter. Leukemia. 2006;20(6):1173-1175. 
457

458

21. H J, J D, X H, et al. Genetic variants in de novo lipogenic pathway genes predict the prognosis of surgicallytreated hepatocellular carcinoma. Sci Rep-Uk. 2015;5:9536.

22. Tao Q, Huang H, Geiman TM, et al. Defective de novo methylation of viral and cellular DNA sequences in ICF syndrome cells. Hum Mol Genet. 2002;11(18):2091-2102.

23. Ying J, Li H, Cui Y, Wong AHY, Langford C, Tao Q. Epigenetic disruption of two proapoptotic genes MAPK10/JNK3 and PTPN13/FAP-1 in multiple lymphomas and carcinomas through hypermethylation of a common bidirectional promoter. Leukemia. 2006;20(6):1173-1175.

24. Hayslip J, Montero A. Tumor suppressor gene methylation in follicular lymphoma: a comprehensive review. Mol Cancer. 2006;5:44.

25. Jones PA, Baylin SB. The fundamental role of epigenetic events in cancer. Nat Rev Genet. 2002;3(6):415-428.

26. Luo C, Hajkova P, Ecker JR. Dynamic DNA methylation: In the right place at the right time. Science. 2018;361(6409):1336-1340.

27. Dor Y, Cedar H. Principles of DNA methylation and their implications for biology and medicine. Lancet. 2018;392(10149):777-786.

28. Tao Q, Chan AT. Nasopharyngeal carcinoma: molecular pathogenesis and therapeutic developments. Expert Rev Mol Med. 2007;9(12):1-24.

29. Rashid A, Issa JP. CpG island methylation in gastroenterologic neoplasia: a maturing field. Gastroenterology. 2004;127(5):1578-1588.

30. Xiang TX, Yuan Y, Li LL, et al. Aberrant promoter CpG methylation and its translational applications in breast cancer. Chin J Cancer. 2013;32(1):12-20.

31. Barbano R, Muscarella LA, Pasculli B, et al. Aberrant Keap1 methylation in breast cancer and association with clinicopathological features. Epigenetics-Us. 2013;8(1):105-112.

32. Kazanets A, Shorstova T, Hilmi K, Marques M, Witcher M. Epigenetic silencing of tumor suppressor genes: Paradigms, puzzles, and potential. Biochim Biophys Acta. 2016;1865(2):275-288.

33. Herath NI, Leggett BA, MacDonald GA. Review of genetic and epigenetic alterations in hepatocarcinogenesis. J Gastroenterol Hepatol. 2006;21(1 Pt 1):15-21.

34. Herceg Z, Paliwal A. Epigenetic mechanisms in hepatocellular carcinoma: how environmental factors influence the epigenome. Mutat Res. 2011;727(3):55-61.

35. Yanase N, Hata K, Shimo K, Hayashida M, Evers BM, Mizuguchi J. Requirement of c-Jun NH2-terminal kinase activation in interferon-alpha-induced apoptosis through upregulation of tumor necrosis factor-related apoptosis-inducing ligand (TRAIL) in Daudi B lymphoma cells. Exp Cell Res. 2005;310(1):10-21.

36. Lohitesh K, Chowdhury R, Mukherjee S. Resistance a major hindrance to chemotherapy in hepatocellular carcinoma: an insight. Cancer Cell Int. 2018;18(1):44.

37. Chen L, Chan HM, Guan XY. Clinical significance of CHD1L in hepatocellular carcinoma and therapeutic potentials of virus-mediated CHD1L depletion. 2011. 
494

495

496

497

498

499

500

501

502

503

504

505

506

507

508

509

510

511

512

513

514

515

516

517

518

\section{Figure legends}

Figure 1 Mapk10 protein expression in HCC specimens shown by immunohistochemistry. Positive Mapk10 staining was observed in adjacent non-tumor tissue. Negative Mapk10 staining in $\mathrm{HCC}$ tissue.

Figure 2 Kaplan-Meier survival curves of HCC (n =59) after gastrectomy.

The survival rate of patients in the group of negative MAPK10 expression was significantly lower than that of patients in the group of positive expression (log-rank test $P=0.008$ ).

Figure 3 Mapk10 mRNA expression in HCC cell lines.

Figure 4 Promoter CpG methylation detection of Mapk10. A. Mapk10 methylation in HCC and adjacent non-tumor tissues. B. Mapk10 methylation in HCC cell lines. C. Mapk10 gene expression in HCC cell lines. (MSP: methylation-specific PCR; M: methylated alleles; U: unmethylated alleles)

Figure 5 Mapk10 expression of HCC cell lines after treatment with TSA and 5-Aza. (MSP: Methylation-specific PCR; M: methylated alleles; U: unmethylated alleles)

Figure 6 MAPK10 inhibits the colony formation of HCC cells. A. The subcellular localization of MAPK10 was observed after $\mathrm{HCC}$ cell lines were transfected with Mapk10 recombinant plasmids. B. MAPK10 protein expression detected by WB. C. Analysis of MAPK10 protein expression. D. Colony formation detection. E. Colony formation efficiency. $(* * P<0.01)$

Figure 7 Ectopic expression of MAPK10 induces apoptosis of HCC cell lines.

A. Apoptosis assay using Annexin V-PE and 7-AAD double staining. B. TUNEL assay. C. Protein level of cleaved-PARP detected by WB after the transduction of Mapk10.

15 Figure $8 M A P K 10$ enhances the chemosensitivity of $\mathrm{HCC}$ cells to 5-FU. A. Cell viability assay 16 of Hep3B after transfected with Mapk10. B. Cell viability assay of HepG2 after transfected with 7 Mapk10. $(* P<0.01, * * P<0.01)$ 
Figure 1

Figure 1 Mapk10 protein expression in HCC specimens shown by immunohistochemistry.

Positive Mapk10 staining was observed in adjacent non-tumor tissue. Negative Mapk10 staining in HCC tissue.

Non-tumor tissue
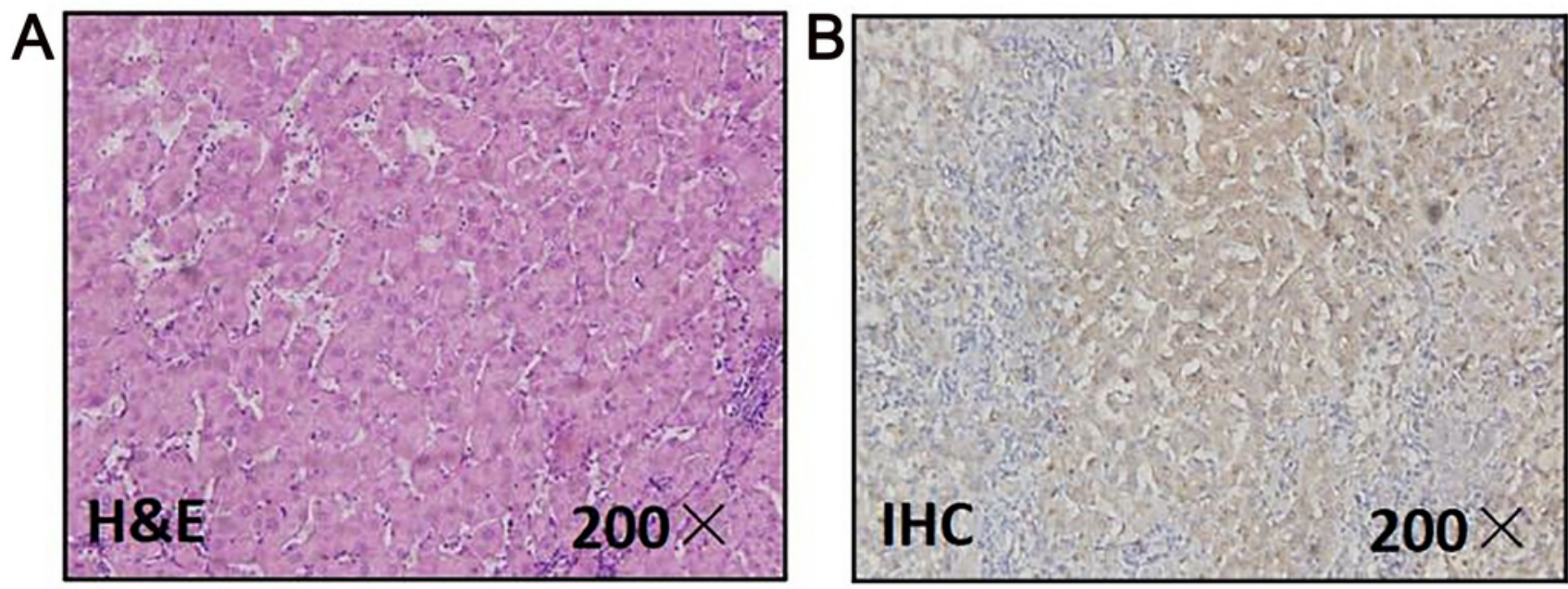

HCC tissue
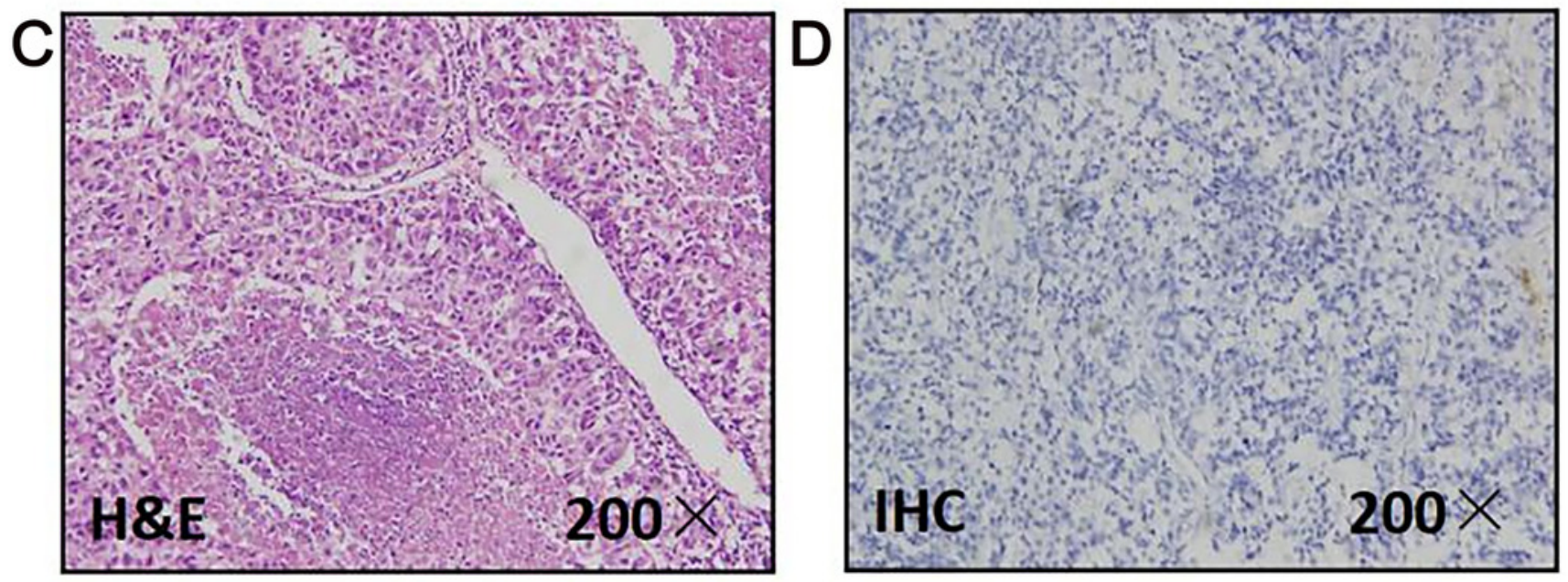
Figure 2

Figure 2 Kaplan-Meier survival curves of HCC ( $n=59)$ after gastrectomy.

The survival rate of patients in the group of negative MAPK10 expression was significantly lower than that of patients in the group of positive expression (log-rank test $P=0.008$ ).

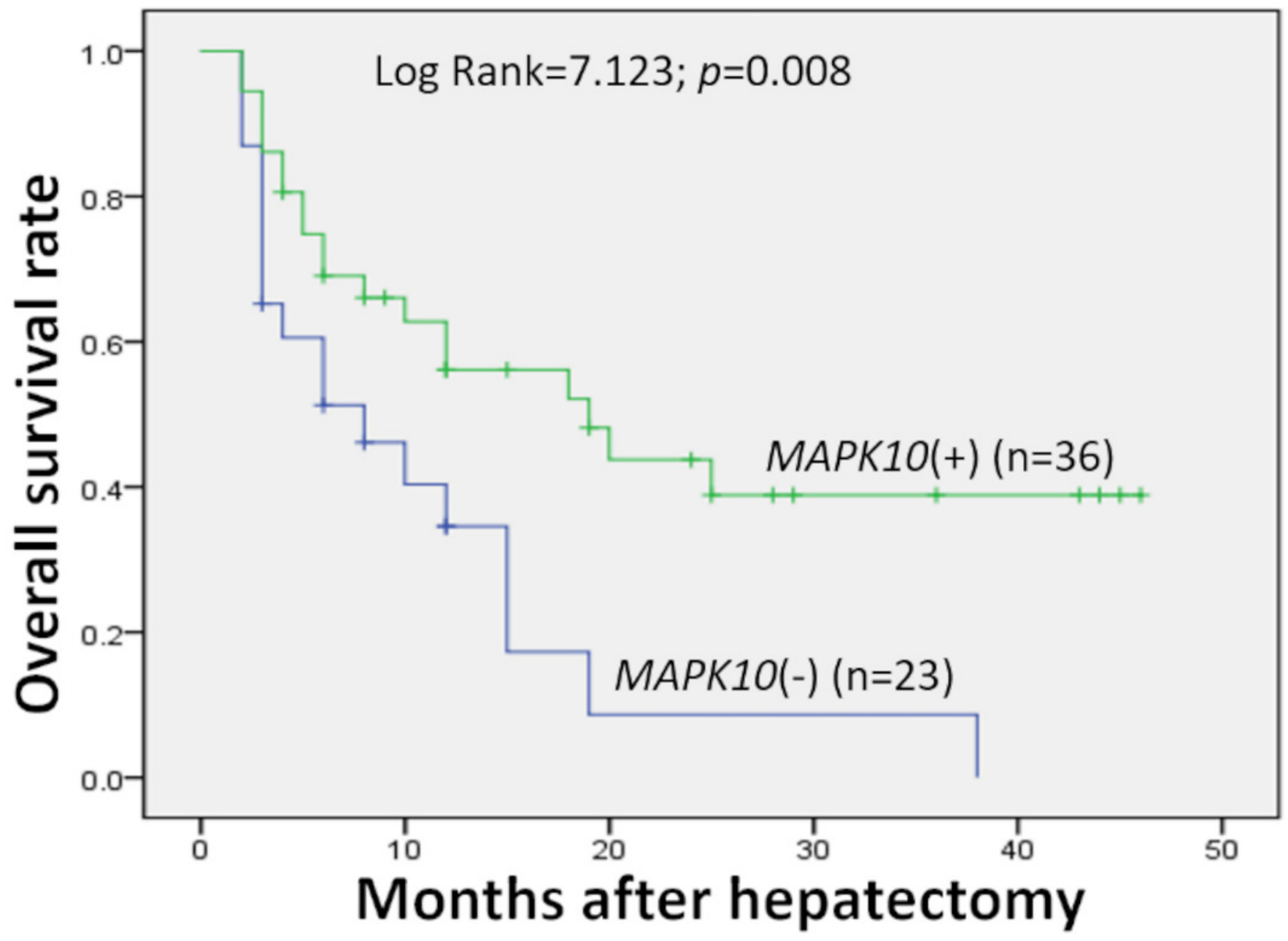


Figure 3

Figure 3 Mapk10 mRNA expression in HCC cell lines.

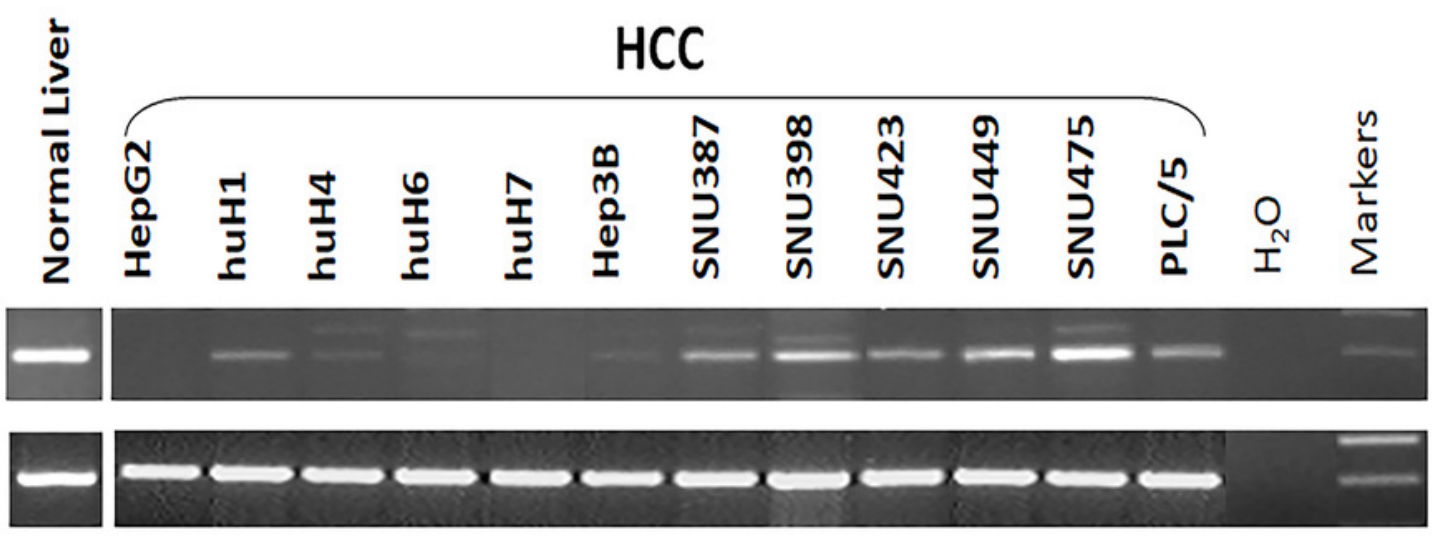


Figure 4

Figure 4 Promoter $\mathrm{CpG}$ methylation detection of Mapk10.

A. Mapk10 methylation in HCC and adjacent non-tumor tissues. B. Mapk10 methylation in HCC cell lines. C. Mapk10 gene expression in HCC cell lines. (MSP: methylation-specific PCR; M: methylated alleles; U: unmethylated alleles)

A

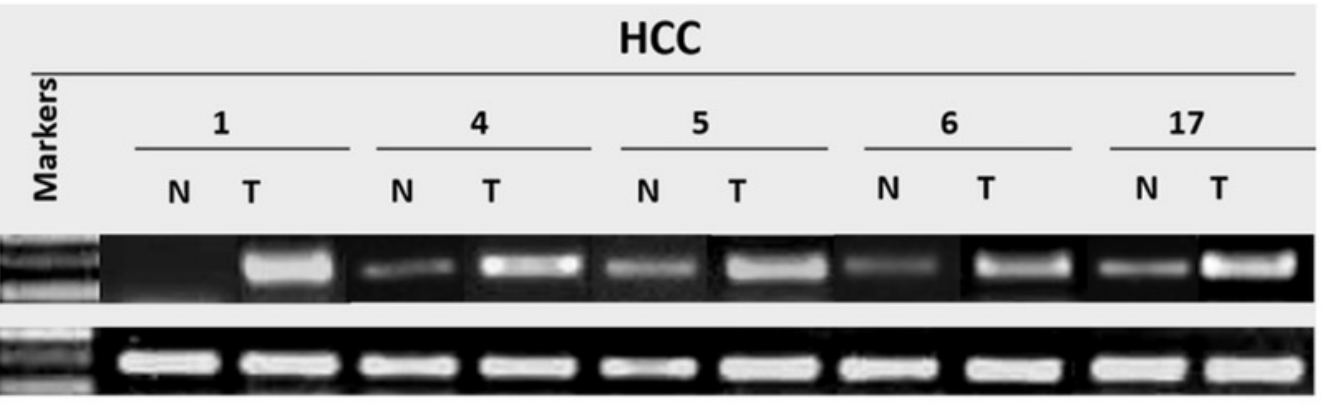

B

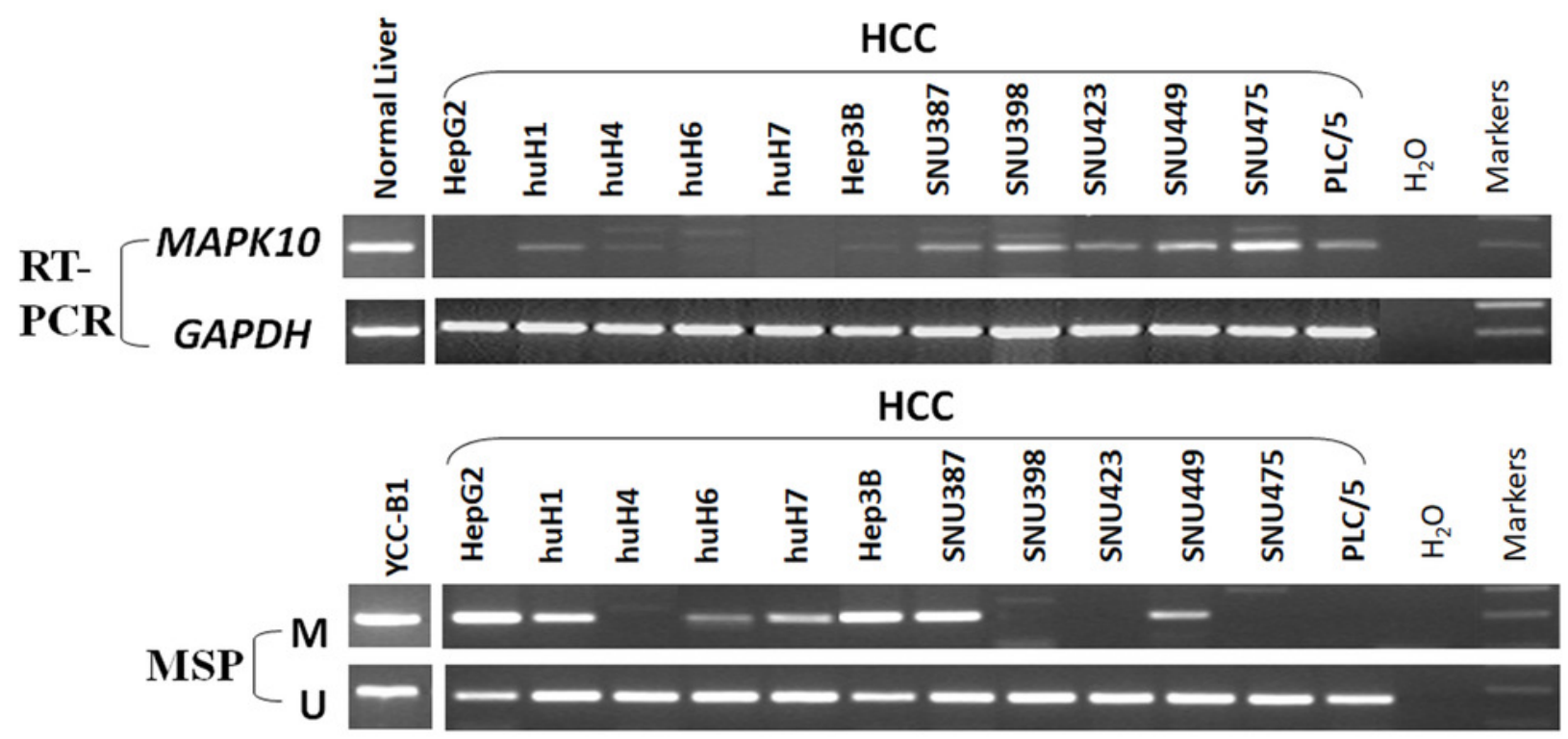

HCC

C 


\section{Figure 5}

Figure 5 Mapk10 expression of HCC cell lines after treatment with TSA and 5-Aza. (MSP: Methylation-specific PCR; M: methylated alleles; U: unmethylated alleles)

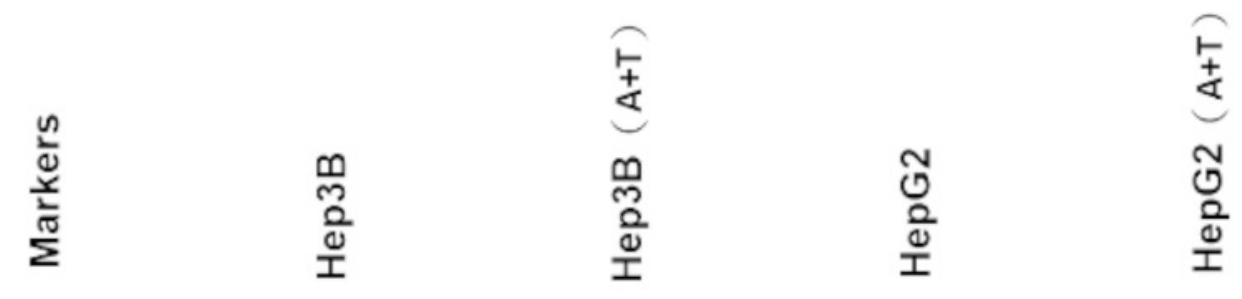

MAPK10

GAPDH

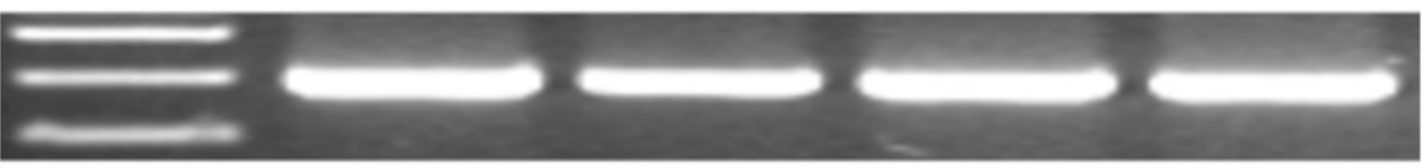

M

MSP

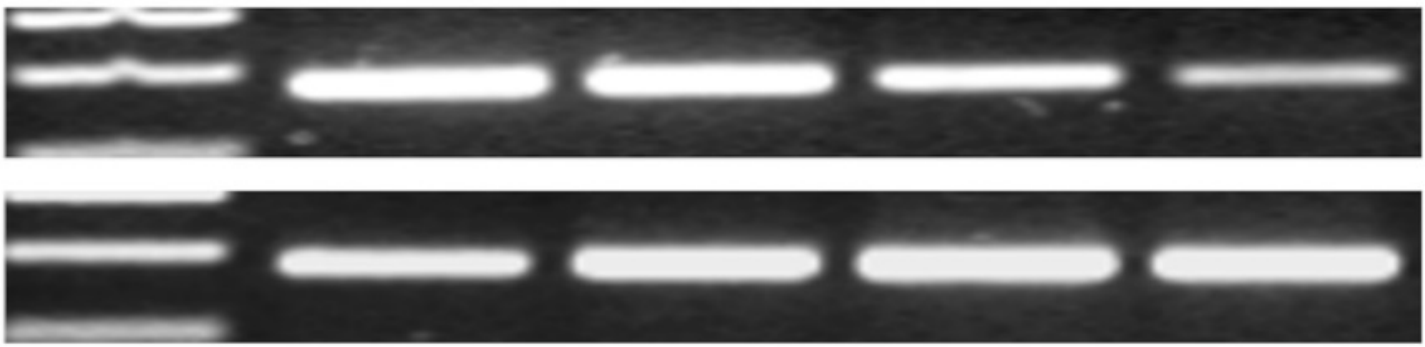


Figure 6

Figure 6 MAPK10 inhibits the colony formation of HCC cells.

A. The subcellular localization of MAPK10 was observed after HCC cell lines were transfected with Mapk10 recombinant plasmids. B. MAPK10 protein expression detected by WB. C. Analysis of MAPK10 protein expression. D. Colony formation detection. E. Colony formation efficiency. $(* * P<0.01)$ 
A

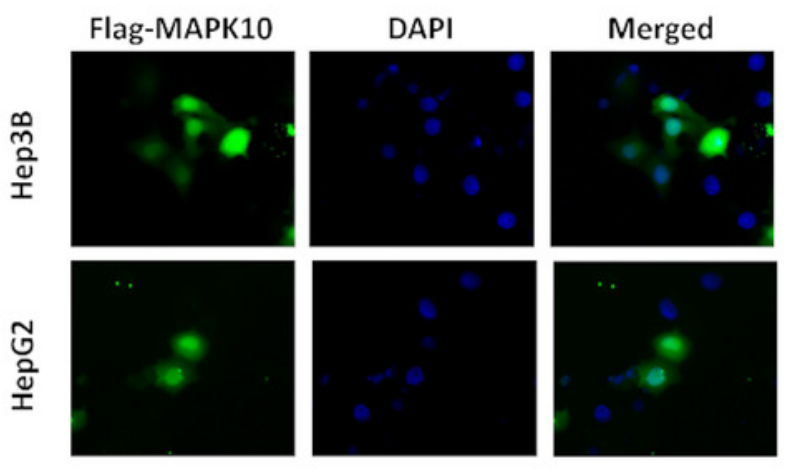

B

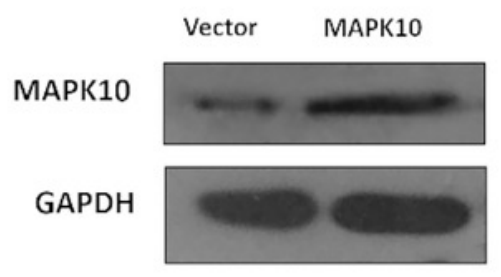

Hep3B

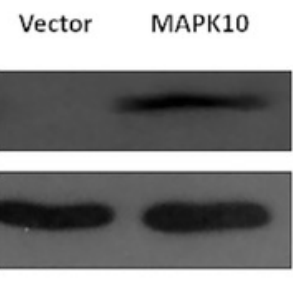

HepG2

C

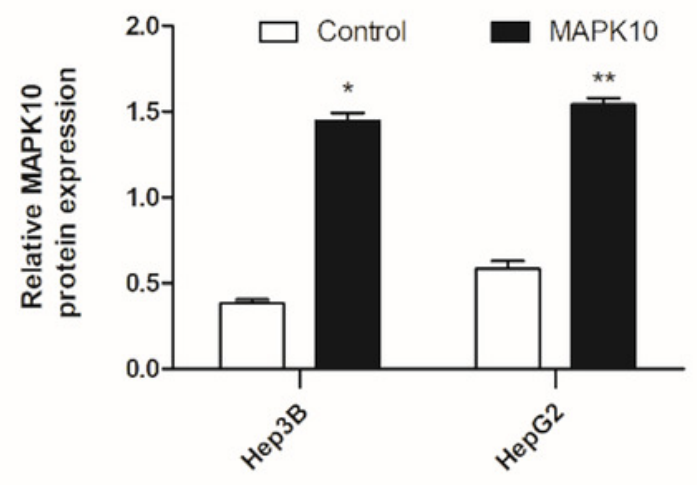

D

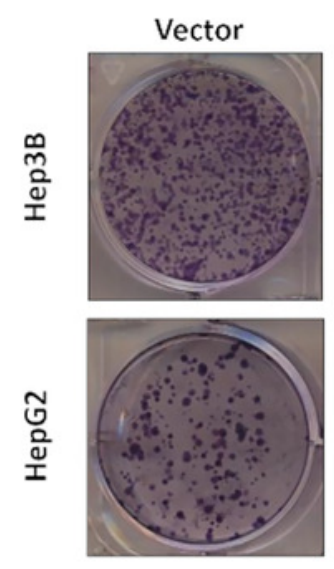

MAPK10
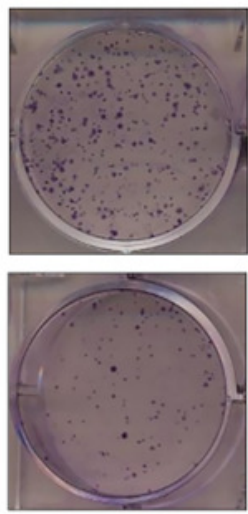

E

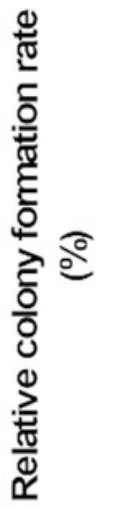




\section{Figure 7}

Figure 7 Ectopic expression of MAPK10 induces apoptosis of HCC cell lines.

A. Apoptosis assay using Annexin V-PE and 7-AAD double staining. B. TUNEL assay. C. Protein level of cleaved-PARP detected by WB after the transduction of Mapk10.

A

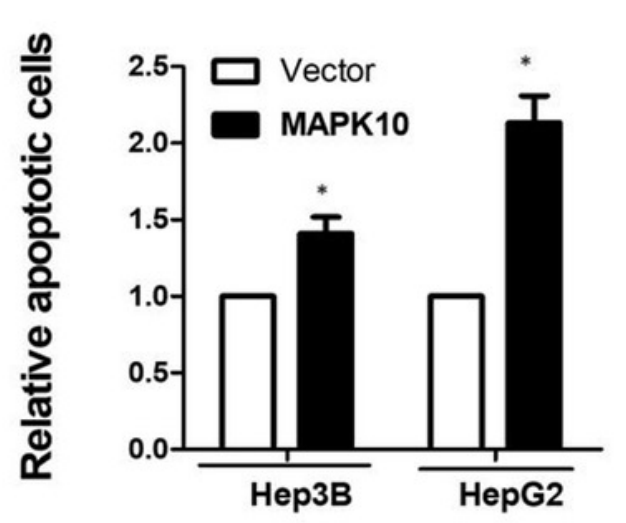

C
B

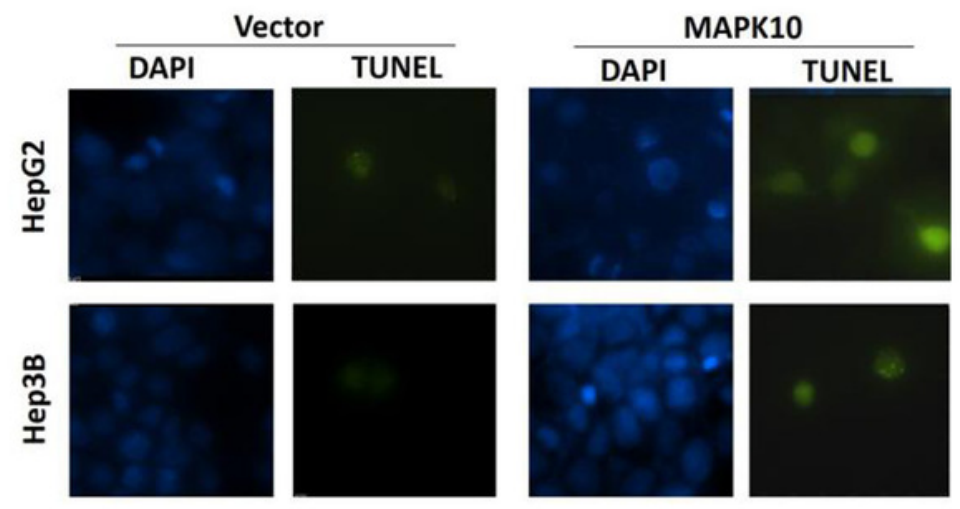

Cleaved-PARP

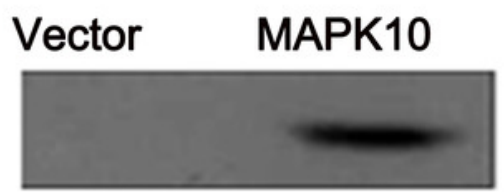

Vector MAPK10

GAPDH

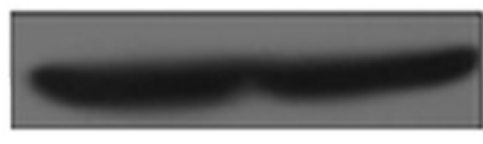

HepG2

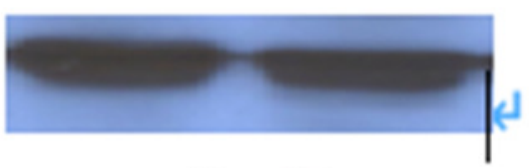

Нер3В 
Figure 8

Figure 8 MAPK10 enhances the chemosensitivity of HCC cells to 5-FU.

A. Cell viability assay of Hep3B after transfected with Mapk10. B. Cell viability assay of HepG2 after transfected with Mapk10. $(* P<0.01, * * P<0.01)$ 
A

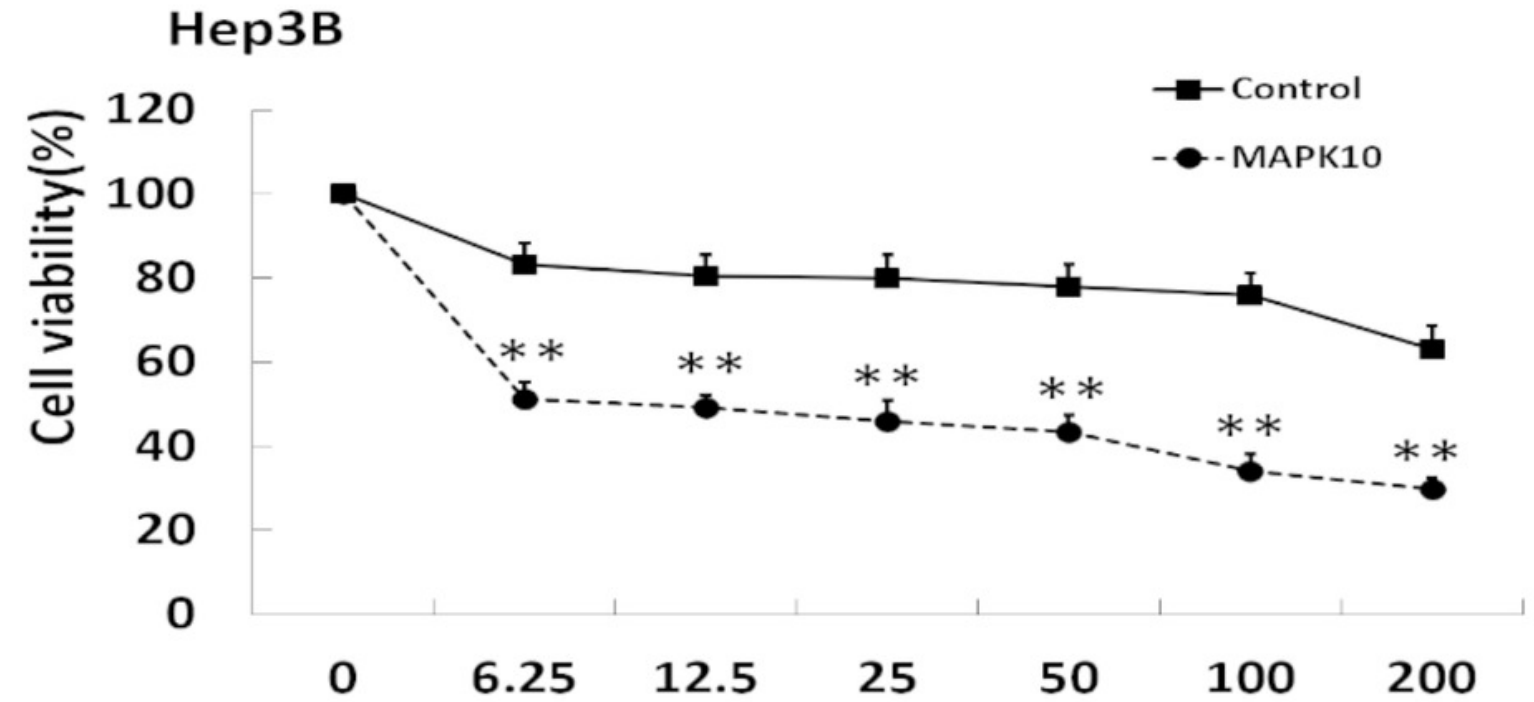

B

\section{HepG2}

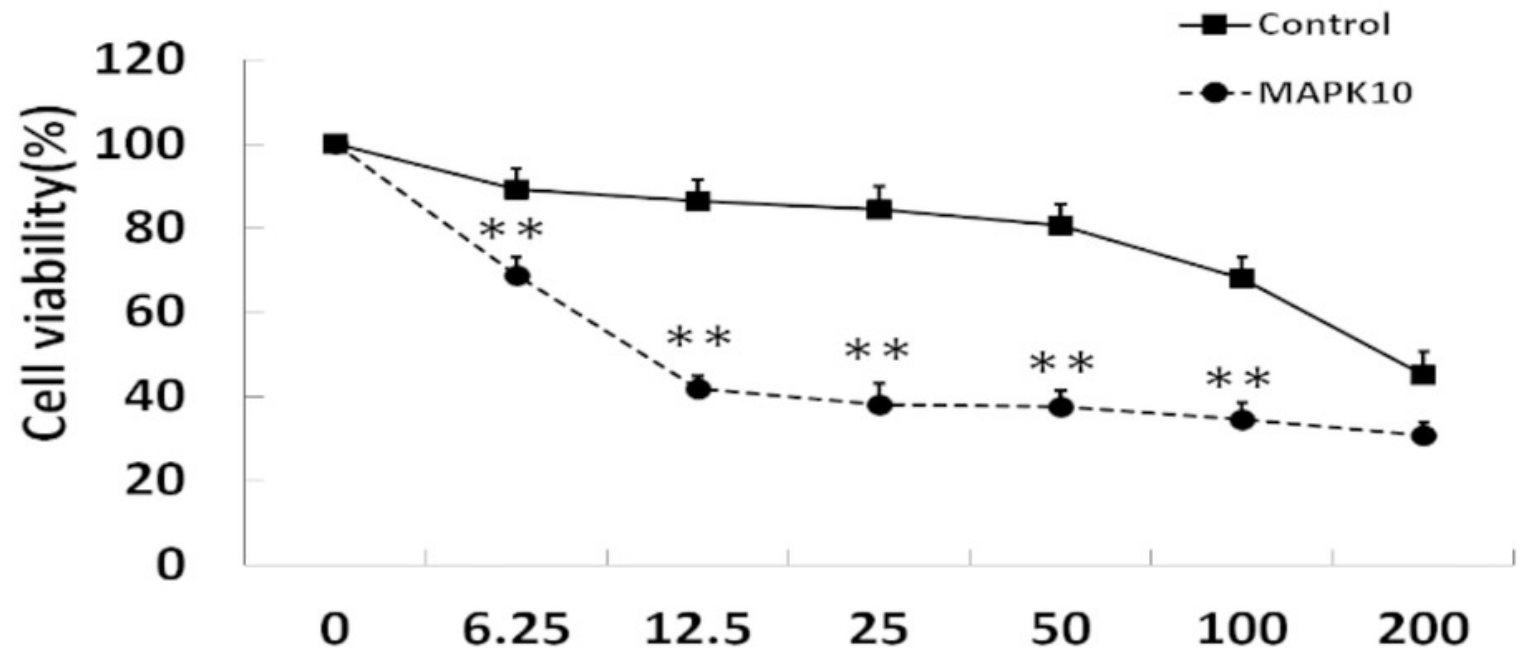




\section{Table $\mathbf{1}$ (on next page)}

Table 1. PCR primer sequences and reaction conditions 
1 Table 1. PCR primer sequences and reaction conditions

\begin{tabular}{|c|c|c|c|c|}
\hline \multirow[t]{4}{*}{ PCR } & Primers & $\mathrm{Siz}$ & TA & PCR \\
\hline & & e & $\left({ }^{\circ} \mathrm{C}\right.$ & cycle \\
\hline & & (bp & ) & $\mathrm{S}$ \\
\hline & & ) & & \\
\hline
\end{tabular}

RT-PCR

MAPK10 F3: 5' -cagctctctaaattgactcag-3'

RR3: 5'-

$248 \quad 55 \quad 32$

ccaatgttggttcactgcag-3'

GAPDH

333: $5^{\prime}-$

gatgaccttgccacagcct-3'

355: $5^{\prime}-$

$\begin{array}{lll}304 & 55 \quad 23\end{array}$

atctctgccctctgctga-3,

Methylation-

specific

PCR (MSP)

Methylated m3: 5' -cgagtagttttagcggttac-3'

$\begin{array}{lll}160 & 60 & 40\end{array}$

m5: 5'-aaaaccttctaacgcgaacga-3'

Unmethylate u3: 5'-tgtgagtagttttagtggttat-3'

$163 \quad 58 \quad 40$

d u5: 5' -caaaaccttctaacacaaacaa-3' 
2 TA, annealing temperature.

3

Peer) reviewing PDF | (2020:03:46905:3:1:NEW 23 Dec 2020) 
Table 2 (on next page)

Table 2. Clinicopathological correlation of Mapk10 protein expression in HCC 
1 Table 2. Clinicopathological correlation of Mapk10 protein expression in HCC

\begin{tabular}{lllll}
$\begin{array}{l}\text { Clinicopathological } \\
\text { parameters }\end{array}$ & number & $\begin{array}{c}\text { Mapk10 } \\
\text { expression }\end{array}$ & p value \\
\hline Features & $(\mathbf{n = 5 9 )}$ & negative & positive & \\
Age & & & & \\
$\leq 60$ & 49 & 18 & 31 & 0.442 \\
$>60$ & 10 & 5 & 5 &
\end{tabular}

Sex

Male

47

17

30

0.389

Female

12

6

6

\section{HBsAg}

Negative

17

6

11

0.717

Positive

42

17

25

Serum AFP(ng/ml)

$\leq 400$

30

8

22

$0.05^{*}$

$>400$

29

15

14

$\operatorname{Tumor} \operatorname{size}(\mathrm{cm})^{\wedge}$ :

$\begin{array}{lllll}\leq 5 & 20 & 5 & 15 & 0.119 \\ >5 & 39 & 18 & 21 & \end{array}$

Microsatellite nodules
Absent
51
17
34
$0.025^{*}$ 


$\begin{array}{llll}\text { Present } & 8 & 6 & 2\end{array}$

\section{Cirhosis}

Absent

Present

PVTT

Absent

Present

47

12

18

29

0.834

5

7

\section{Tumor stage(AJCC)}

Stage I

23

3

20

$0.001^{* *}$

Stage II

7

28

3

4

Stage III

2

$\wedge$ : tumor size was measured by the length of the largest tumor nodule

$3 *$ : Statistically significant $(P<0.05)$.

4

**: Statistically significant $(P<0.01)$.

5 PVTT: Portal vein tumor thrombus

6 AJCC: American Joint Committee on Cancer 